\title{
TWO-POINT OSCILLATIONS IN SECOND-ORDER LINEAR DIFFERENTIAL EQUATIONS
}

\author{
MERVAN Pašić AND JAMES S. W. WONG
}

Abstract. A second-order linear differential equation $(P): y^{\prime \prime}+f(x) y=0, x \in I$, where $I=$ $(0,1)$ and $f \in C(I)$, is said to be two-point oscillatory on $I$, if all its nontrivial solutions $y \in C(\bar{I}) \cap C^{2}(I)$, oscillate both at $x=0$ and $x=1$, i.e. having sequences of infinite zeros converging to $x=0$ and $x=1$. It necessarily implies that all solutions $y(x)$ of $(P)$ must satisfy the Dirichlet boundary conditions and that $f(x)$ must be singular at both end points of $\bar{I}$. We first describe a class of two-point oscillatory equations of $(P)$. Secondly, we prove that $(P)$ is two-point oscillatory if $f(x)$ satisfies certain Hartman-Wintner type asymptotic conditions. Furthermore, we study the arclength of the graph $G(y)$ of solutions curve $y(x)$ on $I$. Two-point oscillatory equation $(P)$ is said to be two-point rectifiable (unrectifiable) oscillatory if the arclengths of all solutions are finite (infinite). We give conditions on $f(x)$ which imply $(P)$ is two-point rectifiable (unrectifiable) oscillatory. When $(P)$ is two-point unrectifiable oscillatory, we determine the fractal dimension of its solution curves for a special class of $f(x)$ similar to the Euler type equations when $f(x)$ is only singular at one end point of $I$. Finally, the preceding results motivate a study on two-sided oscillations of $(P)$ at an interior point of $\bar{I}$.

Mathematics subject classification (2000): 26A27, 26A45, 28A75, 28A80, 34B05, 34C10.

Keywords and phrases: Linear, singular, Dirichlet boundary value problem, oscillations, graph, rectifiability, fractal dimension, Minkowski content, chirp-like asymptotic behaviour.

\section{REFERENCES}

[1] M. Aigner And G. M. Ziegler, Proofs from THE BOOK, Second Edition Springer-Verlag, BerlinHeidelberg-New York, 1998.

[2] J. Banks, V. Dragan, And A. Jones, Chaos. An Mathematical Introduction., Cambridge, 2003.

[3] L. Caffarelli, R. Kohn, And L. Nirenberg, Partial regularity of suitable weak solutions of the Navier-Stokes equations, Comm. Pure Appl. Math., 35 (1982), 771-831.

[4] W. A. Coppel, Stability and asymptotic behavior of differential equations, D. C. Heath and Co., Boston, Mass., 1965.

[5] L. C. Evans And R. F. Gariepy, Measure Theory and Fine Properties of Functions, CRC Press, New York, 1999.

[6] K. FAlCONER, Fractal Geometry. Mathematical Fondations and Applications, John Willey-Sons, 1999.

[7] P. Hartman, Ordinary Differential Equations, Second edition, Birkhauser, Boston, Basel, Stuttgart, 1982.

[8] M. K. Kwong, M. PašIĆ, And J. S. W. Wong, Rectifiable Oscillations in Second Order Linear Differential Equations, J. Differential Equations, 245 (2008), 2333-2351.

[9] F.-H Lin, A new proof of the Caffarelli-Kohn-Nirenberg theorem, Comm. Pure Appl. Math., 51 (1998), 241-257.

[10] P. Mattila, Geometry of Sets and Measures in Euclidean Spaces. Fractals and rectifiability, Cambridge, 1995.

[11] M. PAŠIĆ, Minkowski-Bouligand dimension of solutions of the one-dimensional p-Laplacian, J. Differential Equations, 190 (2003), 268-305. 
[12] M. PAŠIĆ, Rectifiable and unrectifiable oscillations for a class of second-order linear differential equations of Euler type, J. Math. Anal. Appl., 335 (2007), 724-738.

[13] M. PAšIĆ, Fractal oscillations for a class of second-order linear differential equations of Euler type, J. Math. Anal. Appl., 341 (2008), 211-223.

[14] M. PAšIć, Rectifiable and unrectifiable oscillations for a generalization of the Riemann-Weber version of Euler differential equations, Georgian Math. J., 15 (2008), 759-774.

[15] M. PAšść AND J. S. W. Wong, Rectifiable oscillations in second-order half-linear differential equations, Annali di matematica pura ed applicata, to appear.

[16] M. PAŠIĆ, D. ŽuBRINIĆ, AND V. ŽUPANOVIĆ, Oscillatory and phase dimensions of solutions of some second-order differential equations, Bull. Sci. Math., to appear.

[17] G. Polya And G. Szego, Problems and Theorems in Analysis Vol. I, Springer-Verlag, BerlinHeidelberg-New York, 1972, Reprint 1998.

[18] H. O. Peitgen, H. JÛ́Rgens, And D. Saupe, Chaos and Fractals. New Frontiers of Science, Springer-Verlag, 1992.

[19] V. SCHEFFER, Turbulence and Hausdorf dimension, Lecture Notes in Mathematics No. 565, SpringerVerlag, Berlin, 1976, 94-112.

[20] J. SugIE AND T. HARA, Nonlinear oscillation of second order differential equations of Euler type, Proc. Amer. Math. Soc., 124 (1996), 3173-3181.

[21] J. SUGIE AND K. KITA, Oscillation criteria for second order nonlinear differential equations of Euler type, J. Math. Anal. Appl., 253 (2001), 414-439.

[22] C. A. SwAnson, Comparison and Oscillation Theory of Linear Differential Equations, Acad. Press, New York, 1968.

[23] C. TRICOT, Curves and Fractal Dimension, Springer-Verlag, 1995.

[24] J. S. W. WONG, Oscillation theorems for second order nonlinear differential equations of Euler type, Methods and Applications of Analysis, 3 (1996), 476-485.

[25] J. S. W. Wong, On rectifiable oscillation of Euler type second order linear differential equations, E. J. Qualitative Theory of Diff. Equ., 20 (2007), 1-12.

[26] J. S. W. WonG, On rectifiable oscillation of Emden-Fowler equations, Mem. Differential Equations Math. Phys., 42 (2007), 127-144.

[27] J. S. W. Wong, On rectifiable oscillation of Emden-Fowler equations II., Mem. Differential Equations Math. Phys., to appear. 